INPLASY

PROTOCOL

To cite: Gu et al. High Flow

Nasal Oxygen versus

Conventional Oxygen Therapy

in Gastrointestinal Endoscopy

with Conscious Sedation: A

Systematic Review and Meta-

Analysis. Inplasy protocol

202210022. doi:

10.37766/inplasy2022.1.0022

Received: 06 January 2022

Published: 06 January 2022

Corresponding author:

Wan-Jie Gu

wanjiegu@hotmail.com

Author Affiliation:

Nanjing Drum Tower Hospital,

The Affiliated Hospital of

Nanjing University Medical

School.

Support: None.

Review Stage at time of this submission: Completed but not published.

\section{High Flow Nasal Oxygen versus Conventional Oxygen Therapy in Gastrointestinal Endoscopy with Conscious Sedation: A Systematic Review and Meta-Analysis}

Gu, WJ1; Wang, HT2; Huang, J3; Zhao, ZM4; Zhang, CD55.

Review question / Objective: To compare the efficacy of high flow nasal oxygen with conventional oxygen therapy to prevent hypoxemia in gastrointestinal endoscopy with conscious sedation.

Condition being studied: High flow nasal oxygen, a novel technique, may be an alternative to conventional oxygen therapy. High flow nasal oxygen can deliver heated and humidified oxygen via special nasal cannula with high flow (up to $70 \mathrm{~L} / \mathrm{min}$ ). It has been applied to improve oxygenation in clinical entities, favored by increasing evidence supporting its efficacy. Recently, the use of high flow nasal oxygen has spreaded to gastrointestinal endoscopy. However, the efficacy of high flow nasal oxygen in gastrointestinal endoscopy has not yet been well evaluated due to small sample size of the individual study and conflicting results.

INPLASY registration number: This protocol was registered with the International Platform of Registered Systematic Review and Meta-Analysis Protocols (INPLASY) on 06 January 2022 and was last updated on 06 January 2022 (registration number INPLASY202210022).

Conflicts of interest:

None declared.

\section{INTRODUCTION}

Review question / Objective: To compare the efficacy of high flow nasal oxygen with conventional oxygen therapy to prevent hypoxemia in gastrointestinal endoscopy with conscious sedation.
Rationale: Gastrointestinal endoscopy procedures are increasingly utilized as diagnostic and therapeutic tools in clinical settings. To improve patient comfort and facilitate procedural conditions, sedation is widely used in gastrointestinal endoscopy. Hypoxemia frequently occurs in gastrointestinal endoscopy with sedation 
and can lead to life-threatening complications or even death, especially in high-risk patients. Regarding that oxygen therapy is the traditional cornerstone in the management of hypoxemia, guidelines from both physicians and anesthesiologists consistently recommend the supplement of oxygen to prevent hypoxemia for all patients, regardless of the levels of sedation. Currently, conventional oxygen therapy, via nasal cannula, face mask, or other devices with low flow system, is the most common method of oxygenation. However, conventional oxygen therapy may be unsatisfactory for those patients at high risk of hypoxemia. Thus, superior oxygen delivery options are urgently warranted.

Condition being studied: High flow nasal oxygen, a novel technique, may be an alternative to conventional oxygen therapy. High flow nasal oxygen can deliver heated and humidified oxygen via special nasal cannula with high flow (up to $70 \mathrm{~L} / \mathrm{min}$ ). It has been applied to improve oxygenation in clinical entities, favored by increasing evidence supporting its efficacy. Recently, the use of high flow nasal oxygen has spreaded to gastrointestinal endoscopy. However, the efficacy of high flow nasal oxygen in gastrointestinal endoscopy has not yet been well evaluated due to small sample size of the individual study and conflicting results.

\section{METHODS}

Search strategy: Search terms included nasal cannula, gastroscopy, colonoscopy, gastrointestinal endoscopy, esophagogastroduodenoscopy, endoscopic retrograde cholangiopancreatography, and their variations.

Participant or population: Adults undergoing gastrointestinal endoscopy with conscious sedation.

Intervention: High flow nasal oxygen.

Comparator: Conventional oxygen therapy.

Study designs to be included: Randomized controlled trials.
Eligibility criteria: Studies were considered eligible if they met the following criteria: 1) population: adults undergoing gastrointestinal endoscopy with conscious sedation; 2) intervention: high flow nasal oxygen; 3) comparison: conventional oxygen therapy; 4) outcome: hypoxemia; and 5) design: randomized controlled trials. We excluded studies enrolling patients with hypoxemia before gastrointestinal endoscopy.

Information sources: PubMed, Embase, and the Cochrane Central Register of Controlled Trials, and reference lists.

Main outcome(s): Hypoxemia.

Additional outcome(s): The lowest oxygen saturation.

Data management: The following information was collected: author, year, country, population, sample size, definition of hypoxemia, and parameters (device, flow, and FiO2). Extracted data were entered into a data extraction form (Excel, Microsoft Corporation, USA).

Quality assessment / Risk of bias analysis: We used the Cochrane Collaboration's tool to assess risk of bias.

Strategy of data synthesis: We calculated risk ratios (RRs) with 95\% confidence intervals (Cls) for dichotomous outcomes and mean differences (MDs) with $95 \%$ Cls for continuous outcomes. We pooled data using random-effects models with the intention-to-treat principle. Statistical heterogeneity across the trials was assessed by the Cochrane $Q$ test (with $P<$ 0.1 indicating significance) and quantified by the 12 statistic ( $12>50 \%$ for significant heterogeneity). Publication bias was assessed by the Begg's and Egger's tests and further validated by constructing funnel plot.

Subgroup analysis: Subgroup analyses were carried out according to the risk of hypoxemia. High or low risk was defined by the investigators in each trial. For example, patients at high risk were those with obese, 
ASA $\geq 3$, or endoscopic retrograde cholangiopancreatography in prone position; otherwise, they were considered to be at low risk.

Sensitivity analysis: To test the robustness of the results, we conducted the following sensitivity analyses: the leave-one-out method (omitting one trial each time and repeating the meta-analysis).

Language: No restriction.

Country(ies) involved: China.

Other relevant information: None.

Keywords: nasal cannula, high flow, gastrointestinal endoscopy, hypoxemia.

Contributions of each author:

Author 1 - Wan-Jie Gu - conceived the study, collected all data, analyzed and interpreted the data, drafted the manuscript, approved the final draft of the manuscript.

Email: wanjiegu@hotmail.com

Author 2 - Hao-Tian Wang - conceived the study, collected all data, drafted the manuscript, approved the final draft of the manuscript.

Email: ht.wang.qmul@outlook.com

Author 3 - Jiao Huang - conceived the study, collected all data, drafted the manuscript, approved the final draft of the manuscript.

Email: hjstet@163.com

Author 4 - Zhe-Ming Zhao - conceived the study, analyzed and interpreted the data, approved the final draft of the manuscript.

Email: calf1986@126.com

Author 5 - Chun-Dong Zhang - conceived the study, analyzed and interpreted the data, approved the final draft of the manuscript.

Email: zhangchundong2007@126.com 